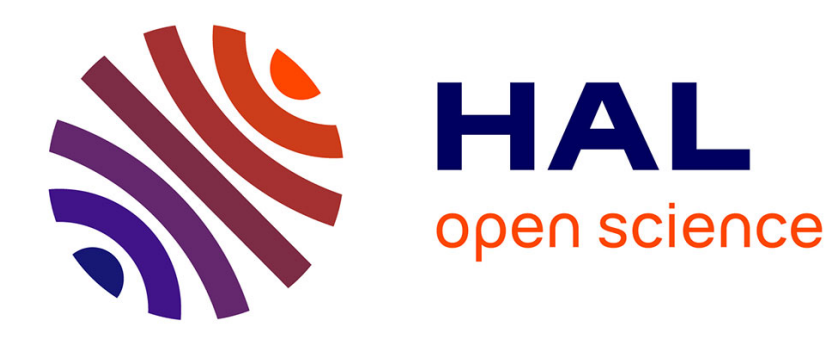

\title{
Méthode multifonction de restauration des enregistrements anciens
}

A. Czyzewski

\section{To cite this version:}

A. Czyzewski. Méthode multifonction de restauration des enregistrements anciens. Journal de Physique IV Proceedings, 1994, 04 (C5), pp.C5-1387-C5-1390. 10.1051/jp4:19945309 . jpa-00253024

\section{HAL Id: jpa-00253024 https://hal.science/jpa-00253024}

Submitted on 1 Jan 1994

HAL is a multi-disciplinary open access archive for the deposit and dissemination of scientific research documents, whether they are published or not. The documents may come from teaching and research institutions in France or abroad, or from public or private research centers.
L'archive ouverte pluridisciplinaire HAL, est destinée au dépôt et à la diffusion de documents scientifiques de niveau recherche, publiés ou non, émanant des établissements d'enseignement et de recherche français ou étrangers, des laboratoires publics ou privés. 


\title{
Méthode multifonction de restauration des enregistrements anciens
}

\section{A. CZYŻEWSKI}

Faculty of Electronics, Sound Engineering Department, Technical University of Gdańsk, 80-952 Gdańsk, Poland

\begin{abstract}
Developments in digital signal processing allow sound engineers to restore old recordings. The elementary tools used in the mentioned processes are digital editing of sound supported by the advanced visualization of the processed signal, equalizing filtering and adaptive digital filtering used to diminish continuous noise. Moreover, various methods of the elimination of impulse noises were tried. Some of the above methods were practically applied to the constructed computer sound restoration system presented in the paper. Consequently, the multi-task sound restoration method was proposed and practically employed. Application of some types of procedures to the sound restoration of old recordings will be discussed. The reduction of background noise using digital filtering in the critical bands was investigated. Another procedure for old magnetic tape echoes cancellation was implemented and tested. Application of smoothing filters to the elimination of noises will be also discussed.
\end{abstract}

\section{INTRODUCTION}

La notion de la restauration des enregistrements phonographiques signifie toute opération exercée sur le signal phonique afin de reconstituer sa forme originale, sans déformations, parasites et bruits qui lui ont été apportés en cours de son enregistrement, sa conservation ou sa reproduction. Les tâches principales de ce type de restauration sont: élimination des distortions harmoniques, détection et élimination des bruits impulsionnels et, enfin, élimination des bruits parasites. Le choix de la méthode déterminée de la suppression de distortions et parasites est toujours soumis aux exigences imposées par le genre du matériel enregistré à reconstituer. Cette présentation servira à montrer les méthodes choisies de restauration des enregistrements sonores utilisées par le logiciel spécialement conçu à ce fin qui est en train d'être élaboré. La description plus précis de méthodes effectuées en même temps que la présentation de principaux résultats obtenus seront montrer pendant la présentation de ce travail.

\section{REDUCTION DES BRUITS PAR FILTRAGE ADAPTATIF}

Le bruit est une distortion qui n'est pas facilement séparable du signal et les essais de son élimination ne donnent pas de résultats satisfaisants à moins que l'on prenne en considération les propriétés du signal dans son domaine temporel et fréquentiel en même temps.

Research sponsored by KBN, Warsaw, Poland. Grant No. \& s 50401005 
Le rapport signal sur bruit peut être exprimé au moyen du coefficient $\sigma_{y}{ }^{2} / \sigma_{x}^{2}$ où $\sigma_{y}^{2}$ signifie la variance du signal et $\sigma_{\mathrm{x}}{ }^{2}$ - celle du bruit. Sur la base de ce coefficient il est possible de déterminer si le bruit entrant a été amplifié ou affaibli après être passé par le filtre de traitement de transmittance égale à $H(z)$. Le dit filtre doit traiter les échantillons disponibles du signal afin d'en éliminer les composants du bruit sans modifier ces du signal. La tâche la plus difficile à affronter se produit dans le cas où le spectre du son et celui du bruit se superposent da façon remarquable. Il est alors très difficile de projeter un filtre vraiment efficace. Dans plusiers cas deux signaux sont disponibles: l'un qui se compose du signal utile mais bruité que nous souhaitons corriger et l'autre qui ne se compose que du bruit perturbateur, corrélé avec les composant du bruit du signal à corriger. La solution trés efficace pour séparation des deux signaux [1] dans ce cas-là est l'application du compensateur adaptatif du bruit, montré sur la Fig. 1.

Pour un filtrage plus précise du signal dans le domaine temporel il est conseillé d'utiliser un filtre inverse dont les coefficients sont calculés sur la base des propriétés statistiques du bruit du signal. Si on suppose que le bruit qui accompagne l'enregistrement à traiter est plus ou moins stationnaire il sera suffisant de prendre en considération le fragment du bruit prélevé, par example, pendant des instants de silence entre deux fragments de musique sur le disque. $\AA$ la sortie du filtre inverse on reçoit alors ${ }^{\star \star}$ un signal où l'énergie du bruit est remarquablement reduite et le spectre de ce bruit ressemble celui blanc.

L'activité suivante qui méne à l'amélioration de la précision de filtrage est une décomposition spectrale du signal traité. Pour effectuer la décomposition du signal on propose un banc de filtres, dont la quantité de filtres et la largeur de leurs bandes passantes sont choisies de façon à adapter le caractère du filtrage aux propriétés de l'oreille humaine. La décomposition spectrale permet de contrôler le processus du traitement du signal séparément dans chaque bande. Cette solution permet de réaliser la réduction des bruits en fonction de la fréquence. L'élément de base dans le système de reduction du bruit de fond est le filtre adaptatif de Wiener à réponse impulsionnelle fini en forme de "lattice". Le principe de fonctionnement de ce système consiste à éliminer les corrélations entre signaux $e_{n}$ et $y_{n}$. Si ces signaux sont corrélés de façon quelconque le filtre répondra avec l'adaptation des coefficients jusqu'au moment où une telle corrélation sera éliminée de la sortie $e_{n}$. Ceci est dû au fait que le filtre adaptatif produit le meilleure réplique possible $d u$ bruit présent dans le signal $x_{n}$ qui est ensuite éliminée de ce signal. Donc le signal de sortie $e_{n}$ se composera principalement du signal que l'on souhaite reconstituer.

En ce qui concerne la méthode du filtrage effectuée, on a pris en compte les aspects perceptuels basés sur l'implémentation du modèle des bandes critiques et on a constaté que cette approche est mieux adaptée au probléme de reduction des bruits.

\section{ELIMINATION DES ECHOS PARASITES}

Le problème essentiel de la reconstruction des enregistrements anciens est celui des échos parasites dont l'origine est constituée par l'effet du copiage de l'enregistrement magnétique des vieilles bandes. Les systèmes homomorphiques par rapport à l'opération de la convolution utilisant les méthodes de l'élimination de ce type de distortions.

Le signal qui est en effet la somme du signal utile et de ses répliques retardées et multipliées par les coefficients déterminés peut être présenté en forme de la formule suivante:

$$
x(n)=s(n)+\sum_{k=1}^{M} \alpha_{k} s\left(n-n_{k}\right)
$$

Le signal peut également être représenté en tant que la convolution du signal de base et du signal parasite: 


$$
\begin{aligned}
x(n) & =s(n) * p(n) \\
\text { où: } p(n) & =\delta(n)+\sum_{k=1}^{M} \alpha_{k} \delta(n-n k)
\end{aligned}
$$

Si l'opération par logarithmotechnie des transformées complexes du signal $x(n)$ a lieu le résultat obtenu prendra la forme des composant additifs correspondant au signal utile $\underline{S}\left(\mathrm{ej}^{\mathrm{j} \omega}\right)$ et au signal parasite $\underline{\mathrm{P}}\left(\mathrm{e}^{\mathrm{j} \omega}\right)$. Le signal correspondant au signal parasite prend alors la forme suivante:

$$
\underline{P}\left(e^{j \omega}\right)=\log \left(1+\alpha_{1} e^{j \omega n 1}\right)
$$

Si la condition qui dit la composante $S\left(\mathrm{e}^{\mathrm{j}(\theta)}\right)$ liée au signal utile sera suffisamment invariante dans le temps par rapport à la composante $\mathrm{P}\left(\mathrm{e}^{\mathrm{j} \omega}\right)$ est remplie la séparation de ces deux composantes au moyen du filtre variant deviendra possible. Ce problème a déjà été présente dans la littérature [2]. L'auteur a décidé de mettre cette méthode dans l'ensemble du logiciel de restauration numérique des enregistrements anciens qu'il est en train d'élaborer, après l'avoir soumise à des modifications adéquates qui ont eu pour l'amélioration de son éfficacité. Ces problèmes seront détailler pendant la présentation orale.

\section{ELIMINATION DES BRUITS IMPULSIONNELS}

La détérioration du support des disques phonographiques est la source des défauts les plus courants des vieux enregistrements. L'un d'eux est le phénomène des parasites impulsionnels ou, simplement, bruits. Dans le cas où ces bruits sont fréquents et leur niveau se rapproche à celui du signal utile leur élimination au moyen des éditeurs du son devient inefficace. Dans une telle situation il faut la méthode de la détection et la correction automatiques des parasites de ce type.

L'autre algorithme de redressement du son, prouvé pratiquement par l'auteur, est un certain "lissage" du spectre au moyen des polynômes approximants. Cette méthode se base sur l'approximation des séquences du signal au moyen des polynômes et le contrôle de l'ordre du polynôme approximant sur la base de l'examen de la valeur de l'erreur moyenne quadratique de l'approximation. Pour estimer au mieux une erreur entre le vecteur des échantillons du signal et le vecteur résultant d'approximation on calcule une métrique d'Euclides défini par:

$$
e_{w}(\bar{Y}, \bar{X})=\left\{\sum_{r=1}^{r}\left(Y_{r}-X_{r}\right)^{2}\right\}^{1 / 2}
$$

où: $\mathrm{Y}_{\mathrm{r}}$ - un élément du vecteur d'approximation $\overline{\mathrm{Y}}$

$X_{r}$ - un élément du vecteur des échantillons du signal $\bar{X}$

Une procédure de contrôle de la relation entre la valeur de l'erreur $e_{w}$ et la longueur $r$ du vecteur $\bar{Y}$ influence alors l'effet de l'opération de redressement du signal. Un exemple de reconstitution du signal à support de la méthode des polynômes approximants est illustré en Fig. 2, notons que les valeurs correspondantes de la longueur du vecteur et de l'ordre de polynôme approximant sont suivantes: 128 et respectivement 6 .

Les meilleurs résultats ont été obtenus par l'auteur avec les algorithmes de l'intelligence artificielle utilisés pour la détection des bruits impulsionnels. Les résultats de ces recherches ont été présentés dans autre publication [3]. 


\section{CONCLUSION}

Les algorithmes du filtrage numérique adaptatif de reduction des bruits, les algorithmes de redressement du signal qui ont été élaborés ainsi que les algorithmes de l'élimination des bruits parasites en forme de l'écho seront réunis dans un logiciel intégré fonctionnant en mode de conversation qui, après avoir définitivement terminé son démarrage, permettra de réaliser une restauration des archives d'enregistrements phoniques.

\section{BIBLIOGRAPHIE}

[1] Varga I., "Adaptive Filtering for Noise Reduction in Audio Signals", Audio Engineering Society Convention, Vienna March 1992 preprint No. 3247.

[2] Oppenheim A., Applications of digital signal processing (Prentice-Hall, Cambridge,1978).

[3] Czyzewski A.and Supron C., "Learning Algorithms for the Cancellation of Old Recordings Noise", Audio Engineering Society Convention, Amsterdam February 1993 (in printing).

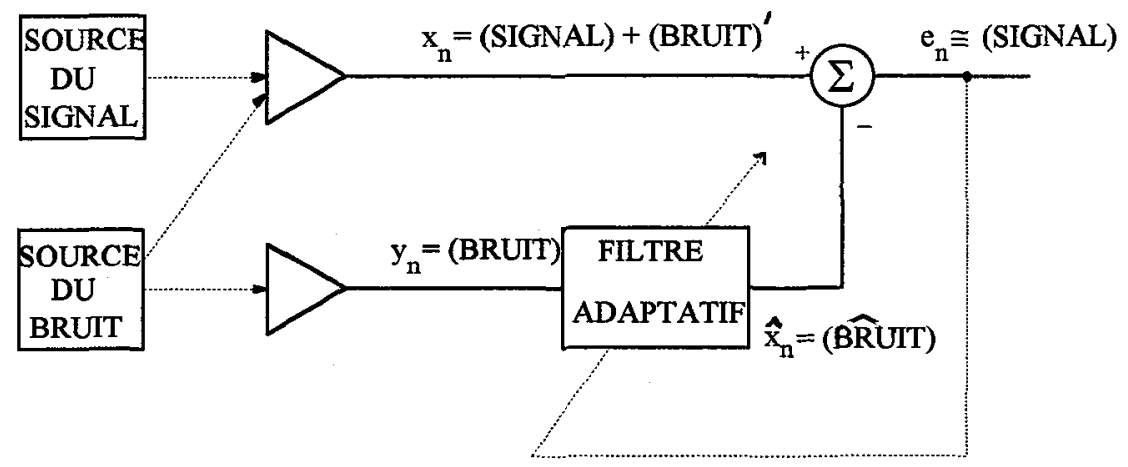

Fig. 1 Principe de la séparation du signal des bruits au moyen du filtre adaptatif.

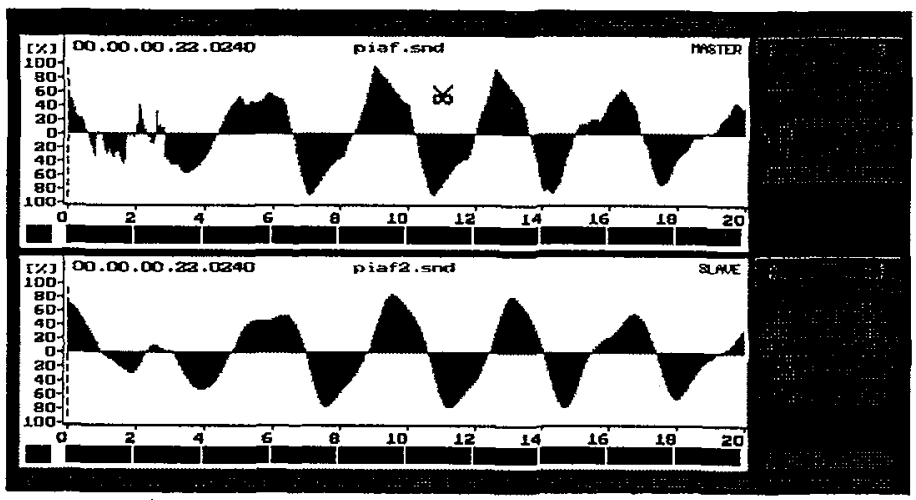

Fig. 2 L'effet de l'opération de redressement du signal à l'aide de l'approximation des polynômes. 